\title{
A GPU-based preconditioned Newton-Krylov solver for flexible multibody dynamics
}

\author{
Radu Serban ${ }^{1 *}$, Daniel Melanz ${ }^{1}$, Ang $\mathrm{Li}^{2}$, Ilinca Stanciulescu ${ }^{3}$, Paramsothy \\ Jayakumar $^{4}$, Dan Negrut ${ }^{1,2}$ \\ ${ }^{1}$ Mechanical Engineering, University of Wisconsin-Madison, Madison, WI 53706 \\ ${ }^{2}$ Electrical and Computer Engineering, University of Wisconsin-Madison, Madison, WI 53706 \\ ${ }^{3}$ Civil and Environmental Engineering, Rice University, Houston, TX 77005 \\ ${ }^{4}$ U.S. Army TARDEC, Warren, MI 48397
}

\begin{abstract}
SUMMARY
This paper describes an approach to numerically approximate the time evolution of multibody systems with flexible (compliant) components. Its salient attribute is that at each time step both the formulation of the system equations of motion and their numerical solution are carried out using parallel computing on Graphics Processing Unit (GPU) cards. The equations of motion are obtained using the Absolute Nodal Coordinate Formulation (ANCF), yet any other multibody dynamics formalism would fit equally well the overall solution strategy outlined herein. The implicit numerical integration method adopted relies on a Newton-Krylov methodology and a parallel direct sparse solver to precondition the underlying linear system. The proposed approach, implemented in a software infrastructure available under an open-source BSD-3 license, leads to improvements in overall simulation times of up to one order of magnitude when compared to matrix-free parallel solution approaches that do not use preconditioning. Copyright (c) 0000 John Wiley \& Sons, Ltd.
\end{abstract}

Received ...

KEY WORDS: inexact Newton; sparse linear system; preconditioning; implicit integration; SPIKE; ANCF; GPU computing

\section{INTRODUCTION}

In multibody dynamics analysis, simulation of compliant yet stiff structures results in numerically stiff systems of differential equations of motion (EOM). Numerical explicit integrators are known to be inefficient on such problems since, for stability reasons, they must use small step sizes - much smaller than dictated by approximation requirements only [1]. Alternatively, implicit integrators can stably solve stiff problems using much larger step sizes. However, the gains obtained from using large integration step sizes come at the price of having to solve at each time step a system of nonlinear algebraic equations. The nonlinear system is typically solved using a Newton-type method [2], which in turn calls for the solution of several linear systems at each integration time step. The lack of an open source parallel sparse linear solver capable of solving these linear systems robustly and efficiently on graphics processing unit (GPU) cards is what motivated an effort whose outcomes are discussed in this paper.

Krylov-subspace iterative methods [3], used to only approximately solve the underlying linear systems and thus rendering the outer loop into an inexact Newton iteration have been

*Correspondence to: 2047 ME, 1513 University Ave., Madison, WI 53706. E-mail: serban@engr.wisc.edu 
shown to be very effective for the implicit integration of stiff differential equations [4]. For good robustness and efficiency, Krylov methods require most of the time a preconditioner. We propose a preconditioner that, just like the rest of the multibody dynamics solution, runs in parallel on the GPU. Specifically, we are introducing a general-purpose, GPU-parallel, preconditioned iterative solver for sparse linear systems. Having the linear solver run on the GPU is essential, since the large number of states typical in flexible multibody dynamics simulations leads to large linear systems whose solution can take advantage of the compute power available on modern GPUs. Herein, the GPU parallel sparse linear solver augments an existing multibody dynamics implementation $[5,6]$ to allow the entire solution sequence to be executed on the GPU. In the process, we show that the preconditioning leads to almost one order of magnitude shorter simulation times.

The open-source software infrastructure that implements this GPU-based solution is called Chrono [7, 8]. Its Chrono: :Flex module provides support for various flexible elements, bilateral constraints (joints), and contact with friction. It offers several solution methods for the resulting equations of motion (EOM) and uses GPU acceleration in both the equation formulation and equation solution stages. For the linear algebra part, the open-source parallel sparse linear solver Spike: : GPU relies on preconditioned Krylov-subspace methods to solve linear systems with tens of thousands of unknowns on the GPU [9]. Spike: : GPU employs a divide-and-conquer approach to factorize banded matrices [10]. For a given sparse linear system, the construction of the preconditioner starts with a preprocessing stage in which a series of reorderings are applied to maximize the absolute values of diagonal entries and minimize bandwidth. The resulting banded matrix is subsequently partitioned into submatrices that are factored in parallel and with no data communication.

The remainder of this paper is structured as follows. Section $\S 2$ provides a brief overview of the formulation of the EOM and the implicit numerical scheme used to solve them. The structure of Spike: :GPU is described in more detail in section $\S 3$. Section $\S 4$ outlines implementation details for both the equation formulation and solution stages of the flexible multibody dynamics problem and the parallel solution of sparse linear systems on the GPU. Results from various numerical experiments that highlight the effectiveness of the proposed solution approach are provided in section $\S 5$. Section $\S 6$ summarizes our findings and outlines directions of future work.

\section{FLEXIBLE MULTIBODY DYNAMICS}

The multibody system dynamics solution calls at each integration time step for performing two tasks: $(i)$ formulating the system EOM, followed by (ii) numerically solving the resulting differential-algebraic equations (DAE). Herein, we use the ANCF approach to formulate the EOM and moreover, we discuss only problems involving gradient deficient ANCF beam elements. The extension to general ANCF elements, while tedious to derive, is straightforward to implement in software and does not change the numerical solution stage, which is the main focus of this contribution. In fact, different choices of flexible multibody formulations can be employed, see for instance [11], as long as an implicit integration formula, which here is chosen to belong to the Newmark family, is used to subsequently discretize the resulting EOM.

\subsection{The Absolute Nodal Coordinate Formulation}

To demonstrate the proposed preconditioned Krylov solver, in this paper we will use gradient deficient ANCF beam elements $[12,13]$ to model flexible multibody systems containing slender beams. Each beam element has two nodes, and each node has associated with it two vector unknowns: one position vector and one gradient vector, i.e., three components of the global position vector of the node, and three components of the position vector gradient at the node, respectively. No shear locking issues are encountered for thin and stiff beams and the reduced number of nodal coordinates leads to a more efficient solution [14]. Note that the gradient 
deficient ANCF beam element cannot describe a rotation of the beam about its own axis, so torsional effects cannot be modeled.

The global position vector of an arbitrary point on the beam centerline with element spatial coordinate $x$ is given by $\mathbf{r}(x, \mathbf{e})=\mathbf{S}(x) \mathbf{e}$, where $\mathbf{e}=\left[\mathbf{e}_{1}^{T}, \mathbf{e}_{2}^{T}\right]^{T} \in \mathbb{R}^{12}$ is the vector of element nodal coordinates. The shape function matrix for this element is defined as $\mathbf{S}=\left[S_{1} \mathbf{I}, S_{2} \mathbf{I}, S_{3} \mathbf{I}, S_{4} \mathbf{I}\right] \in \mathbb{R}^{3 \times 12}$ where $\mathbf{I}$ is the $3 \times 3$ identity matrix; the shape functions $S_{j}, j=1, \ldots, 4$ are defined as [15]

$$
\begin{aligned}
& S_{1}=1-3 \xi^{2}+2 \xi^{3} \\
& S_{2}=\ell\left(\xi-2 \xi^{2}+\xi^{3}\right) \\
& S_{3}=3 \xi^{2}-2 \xi^{3} \\
& S_{4}=\ell\left(-\xi^{2}+\xi^{3}\right),
\end{aligned}
$$

Here, $\xi=x / \ell \in[0,1]$ and $\ell$ is the element length. Kinematic constraints impose restrictions on the relative motion of the bodies in a mechanical system. These constraints are algebraic equations of the form

$$
\Phi(\mathbf{q}, t)=\left[\Phi_{1}(\mathbf{q}, t), \ldots, \Phi_{m}(\mathbf{q}, t)\right]^{T}=\mathbf{0}_{m},
$$

where $m$ is the total number of independent kinematic constraint equations that must be satisfied by the generalized coordinates $\mathbf{q}=\left[\mathbf{q}_{1}^{T} \ldots, \mathbf{q}_{n}^{T}\right]^{T} \in \mathbb{R}^{p}, n$ is the total number of bodies, and $p$ is the total number of coordinates present in the system. The generalized coordinates of the system change in time under the effect of applied forces such that the kinematic constraint equations of Eq. (2) are satisfied at all times. The time evolution of the system is governed by the Lagrange multiplier form of the constrained EOM [16]:

$$
\mathbf{M} \ddot{\mathbf{q}}+\Phi_{\mathbf{q}}^{T}(\mathbf{q}, t) \lambda+\mathbf{Q}^{s}(\mathbf{q})=\mathbf{Q}^{a}(\dot{\mathbf{q}}, \mathbf{q}, t),
$$

where $\mathbf{Q}^{a}$ and $\mathbf{Q}^{s}$ are the generalized applied and elastic forces, respectively, $\Phi_{\mathbf{q}} \in \mathbb{R}^{m \times p}$ is the constraint Jacobian matrix, $\lambda$ is the vector of Lagrange multipliers, and $\mathbf{M} \in \mathbb{R}^{12 \times 12}$ is the symmetric consistent element mass matrix defined in terms of the element mass density and cross sectional area as

$$
\mathbf{M}=\int_{\ell} \rho a \mathbf{S}^{T} \mathbf{S} d x
$$

The generalized element elastic forces are obtained from the strain energy expression [15] as

$$
\mathbf{Q}^{s}=\int_{\ell} \operatorname{Ea\varepsilon }_{11}\left(\frac{\partial \varepsilon_{11}}{\partial \mathbf{e}}\right)^{T} d x+\int_{\ell} E I \kappa\left(\frac{\partial \kappa}{\partial \mathbf{e}}\right)^{T} d x
$$

where $\varepsilon_{11}=\left(\mathbf{r}_{x}^{T} \mathbf{r}_{x}-1\right) / 2$ is the axial strain, $\kappa=\left\|\mathbf{r}_{x} \times \mathbf{r}_{x x}\right\| /\left\|\mathbf{r}_{x}\right\|^{3}$ is the magnitude of the curvature vector, $E$ is the modulus of elasticity, and $I$ is the second moment of area. Since only one spatial coordinate $\xi$ is used in the shape functions, the numerical integration is carried out using the Gauss-quadrature formula in one dimension.

The applied external forces $\mathbf{Q}^{a}$ include gravitational forces and optionally contact and friction forces modeled using a penalty approach [17]. No external forces besides those due to gravity were considered in this work.

\subsection{Implicit Integration}

Equations (2) and (3) form a system of index-3 DAEs [18, 1]. Several low-order numerical integration schemes have been effectively used to solve index-3 DAE [19]. Here we use the Newmark integration scheme [20]; originally used in the structural dynamics community for the numerical integration of a linear set of second order ODEs, it was adapted for the discretization of DAEs [21, 22, 23]:

$$
(\mathbf{M} \ddot{\mathbf{q}})_{n+1}+\left(\Phi_{\mathbf{q}}^{T} \lambda\right)_{n+1}+\left(\mathbf{Q}^{s}-\mathbf{Q}^{a}\right)_{n+1}=\mathbf{0} .
$$


Using the Newmark integration formulas, the position and velocity at a new time step $t_{n+1}$ are obtained in terms of the acceleration $\ddot{\mathbf{q}}_{n+1}$ as

$$
\begin{aligned}
\mathbf{q}_{n+1} & =\mathbf{q}_{n}+h \dot{\mathbf{q}}_{n}+\frac{h^{2}}{2}\left[(1-2 \beta) \ddot{\mathbf{q}}_{n}+2 \beta \ddot{\mathbf{q}}_{n+1}\right] \\
\dot{\mathbf{q}}_{n+1} & =\dot{\mathbf{q}}_{n}+h\left[(1-\gamma) \ddot{\mathbf{q}}_{n}+\gamma \ddot{\mathbf{q}}_{n+1}\right],
\end{aligned}
$$

where $h$ is the integration step size, while $\gamma \geq 1 / 2$ and $\beta \geq(\gamma+1 / 2)^{2} / 4$ are two user selected parameters that control the amount of numerical damping associated with the integration formula. Finally, the new configuration of the system is required to satisfy the kinematic constraint equations at time $t_{n+1}$ :

$$
\Phi\left(\mathbf{q}_{n+1}, t_{n+1}\right)=\mathbf{0} .
$$

This scheme was proved to have convergence order 1 or 2 , depending on the choice of parameters $\gamma$ and $\beta$ [23]. At each time step $t_{n+1}$, the numerical solution begins by solving the nonlinear set of equations (6) and (8) for the unknowns $\ddot{\mathbf{q}}_{n+1}$ and $\lambda_{n+1}$, using Eqs. (7) to eliminate the generalized positions and velocities. This is made clear by rewriting the nonlinear system as $\Psi\left(\ddot{\mathbf{q}}_{n+1}, \lambda_{n+1}\right)=\mathbf{0}_{p+m}$, where

$$
\Psi\left(\ddot{\mathbf{q}}_{n+1}, \lambda_{n+1}\right) \equiv\left[\begin{array}{c}
(\mathbf{M} \ddot{\mathbf{q}})_{n+1}+\left(\Phi_{\mathbf{q}}^{T} \lambda\right)_{n+1}+\left(\mathbf{Q}^{s}-\mathbf{Q}^{a}\right)_{n+1} \\
\Phi\left(\mathbf{q}_{n+1}, t_{n+1}\right) /\left(\beta h^{2}\right)
\end{array}\right] .
$$

The kinematic constraint equations above were scaled to improve the condition number of the Jacobian matrix $[21,24,19]$. The nonlinear algebraic system in Eq. (9) is solved numerically using a Newton-type iterative algorithm. The required Jacobian $\mathbf{J}_{n+1}=$ $\left[\partial \Psi / \partial \mathbf{q}_{n+1}, \partial \Psi / \partial \lambda_{n+1}\right]$ is relatively straightforward to obtain, the most compute intensive term in its evaluation being the partial derivative of the internal forces with respect to the generalized coordinates; i.e., the stiffness matrix $\mathbf{K}^{s} \equiv \partial \mathbf{Q}^{s} / \partial \mathbf{e}$ which can be derived from Eq. (5) as

$$
\begin{aligned}
\mathbf{K}^{s} & =\int_{\ell} E a \varepsilon_{11} \frac{\partial}{\partial \mathbf{e}}\left(\frac{\partial \varepsilon_{11}}{\partial \mathbf{e}}\right)^{T} d x+\int_{\ell} E a\left(\frac{\partial \varepsilon_{11}}{\partial \mathbf{e}}\right)^{T}\left(\frac{\partial \varepsilon_{11}}{\partial \mathbf{e}}\right) d x \\
& +\int_{\ell} E I \kappa \frac{\partial}{\partial \mathbf{e}}\left(\frac{\partial \kappa}{\partial \mathbf{e}}\right)^{T} d x+\int_{\ell} E I\left(\frac{\partial \kappa}{\partial \mathbf{e}}\right)^{T}\left(\frac{\partial \kappa}{\partial \mathbf{e}}\right) d x .
\end{aligned}
$$

Using a Newton-type method, the solution $\left\{\ddot{\mathbf{q}}_{n+1}, \lambda_{n+1}\right\}$ of the nonlinear system in Eq. (9) is obtained iteratively, requiring successive solutions of linear systems of the form

$$
\mathbf{J}_{n+1}^{(k)} \cdot \delta_{n+1}^{(k)}=-\Psi\left(\ddot{\mathbf{q}}_{n+1}^{(k)}, \lambda_{n+1}^{(k)}\right)
$$

to obtain the correction vector $\delta_{n+1}^{(k)}$, where $(k)$ represents a Newton iteration counter. While this method is known to converge quadratically [2], it does so by incurring a great computational cost related to the Jacobian matrix evaluation and factorization at each iteration. Modified Newton methods alleviate this issue by evaluating and factoring the Jacobian only once per time step and reusing it throughout the nonlinear iterations.

\subsection{Newton-Krylov}

Equation (11) defines a modified Newton method as long as a direct linear solver is used to determine the correction $\delta_{n+1}^{(k)}$. Solutions that leverage parallel computing often times rely on Krylov-subspace methods [25], in which $\delta_{n+1}^{(k)}$ is computed iteratively. The resulting inexact Newton iteration has been shown to be effective in the context of implicit integration of stiff differential equations [4]. In this case, the products $\mathbf{J}_{n+1}^{(k)} \mathbf{v}$ are computed in a matrixfree fashion. The term "inexact" indicates that, unlike when a direct solver is used, $\delta_{n+1}^{(k)}$ 
is computed through an iterative process in an inner loop that can be stopped before $\delta_{n+1}^{(k)}$ satisfies Eq. (11) with a high degree of accuracy. This was the approach embraced in [5] using an unpreconditioned BiCGStab linear solver.

Krylov-subspace methods sometimes fail to converge and are almost always inefficient unless an adequate preconditioner is used. A generic linear system $\mathbf{A x}=\mathbf{b}$ can be preconditioned on the left as $\left(\mathbf{P}^{-1} \mathbf{A}\right) x=\mathbf{P}^{-1} \mathbf{b}$, on the right as $\left(\mathbf{A P}^{-1}\right) \mathbf{P} \mathbf{x}=\mathbf{b}$, or on both sides. Here we only consider preconditioning on the left. The Krylov-subspace method is then applied to a system with the modified matrix $\mathbf{P}^{-1} \mathbf{A}$ instead of $\mathbf{A}$. In this paper, we report on a GPU-based general-purpose preconditioner that dovetails with the rest of the equation formulation and solution components of the implementation to produce an entirely GPU-based preconditioned Newton-Krylov solver for flexible multibody dynamics.

\section{PRECONDITIONING}

The preconditioned iterative Krylov-subspace linear solver employed in this work is provided by the Spike: : GPU library [9]. In this section we provide an overview of this package and its use in conjunction with the Chrono: : Flex simulation framework. The discussion will be carried out in the context of solving a generic linear system $\mathbf{A x}=\mathbf{b}, \mathbf{A} \in \mathbb{R}^{N \times N}$, with the understanding that all findings and algorithms are eventually used to solve the linear system in Eq. (11).

\subsection{An overview of Spike::GPU}

The preconditioning methodology implemented in Spike: : GPU for the solution of large-scale sparse linear systems $\mathbf{A x}=\mathbf{b}$ relies on the SPIKE algorithm [26, 10, 27], a divide-and-conquer technique for the solution of banded systems. The salient attribute of this approach is its casting of the preconditioning step as a dense linear algebra problem. Specifically, the preconditioning step consists of the solution of a dense banded linear system with a banded matrix that is obtained from $\mathbf{A}$ after a sequence of reordering strategies and element drop-off. The resulting banded matrix is then partitioned and factorized in parallel with no communication.

3.1.1. Reordering strategies. The approach embraced is predicated on the matrix $\mathbf{A}$ being banded and diagonally dominant. As this is most often not the case, for the solution of general sparse linear systems, an aggressive preprocessing stage is employed to reorder the columns and rows of $\mathbf{A}$. Since $\mathbf{A}$ is not assumed symmetric, no attempt is made to preserve symmetry which adds an extra degree of freedom when a first set of row permutations is applied as $\mathbf{Q A x}=\mathbf{Q b}$, to either maximize the number of nonzero elements on the diagonal of $\mathbf{A}[28]$ or maximize the product of the absolute values of the diagonal entries [29, 30]. Both reordering algorithms are implemented using simple depth first search with a look-ahead technique. In the remainder of this paper, we will refer to this reordering as MC64, from the name of the HSL [31] subroutine that implements this algorithm.

While this first reordering renders QA to be diagonally "heavy", a second reordering, using the traditional Reverse Cuthill-McKee (RCM) algorithm [32], is applied in order to reduce its bandwidth. Since the diagonal entries should not be relocated, the second permutation is applied to the symmetric matrix $\mathbf{Q A}+\mathbf{A}^{T} \mathbf{Q}^{T}$.

Following the reordering, in an attempt to produce an even narrower bandwidth, we drop off elements of the reordered matrix $\mathbf{A}$ that are small in magnitude and far away from the main diagonal. More precisely, we select the smallest half-bandwidth $K$ such that

$$
\sum_{i=1}^{N} \sum_{|j-i| \leq K}\left|a_{i j}\right| \geq \delta \cdot \sum_{i, j=1}^{N}\left|a_{i j}\right|,
$$

where $\delta \in(0,1)$ represents the fraction of the element-wise 1-norm of $\mathbf{A}$ that is retained. 


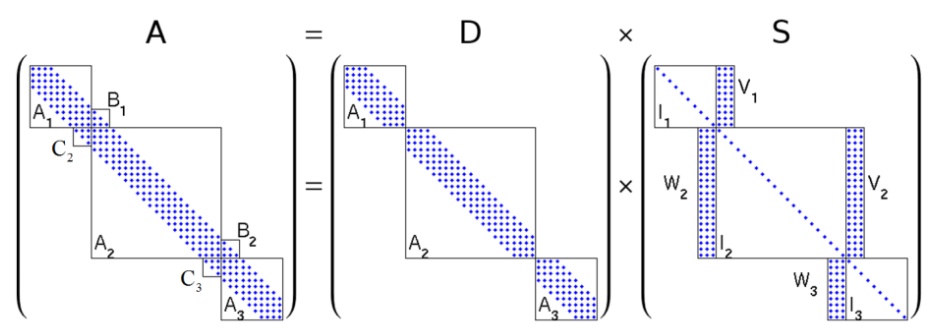

Figure 1. SPIKE factorization of a banded matrix A using $P=3$ partitions.

3.1.2. SPIKE algorithm overview. Assuming that the matrix $\mathbf{A}$ is banded or can be brought to banded form by the process described in $\$ 3.1 .1$, the SPIKE algorithm proceeds with a partitioning of $\mathbf{A}$ into a block tridiagonal form with diagonal blocks $\mathbf{A}_{i}, i=1, \ldots, P$, followed by a factorization of the partitioned matrix into the product between a block diagonal matrix $\mathbf{D}$ and a so-called spike matrix $\mathbf{S}$ as illustrated in Fig. 1. Assuming that each $\mathbf{A}_{i}$ is non-singular, the spike matrices; i.e., the off-diagonal blocks $\mathbf{V}_{i}$ and $\mathbf{W}_{i}$ of $\mathbf{S}$, can be calculated by solving a sequence of independent linear systems [10]:

$$
\begin{aligned}
\mathbf{A}_{1} \mathbf{V}_{1} & =\left[\begin{array}{c}
\mathbf{0} \\
\mathbf{0} \\
\mathbf{B}_{1}
\end{array}\right] \\
\mathbf{A}_{i}\left[\mathbf{W}_{i} \mid \mathbf{V}_{i}\right] & =\left[\begin{array}{cc}
\mathbf{C}_{i} & \mathbf{0} \\
\mathbf{0} & \mathbf{0} \\
\mathbf{0} & \mathbf{B}_{i}
\end{array}\right], i=2, \ldots, P-1 \\
\mathbf{A}_{P} \mathbf{W}_{P} & =\left[\begin{array}{c}
\mathbf{C}_{P} \\
\mathbf{0} \\
\mathbf{0}
\end{array}\right] .
\end{aligned}
$$

With this decomposition, the task of solving the linear system $\mathbf{A x}=\mathbf{b}$ is thus reduced to solving $\mathbf{D g}=\mathbf{b}$, which is trivial and embarrassingly parallel, followed by solving $\mathbf{S x}=\mathbf{g}$. At the cornerstone of SPIKE lies the observation that solving $\mathbf{S x}=\mathbf{g}$ can be further reduced to solving a system of much smaller size, $\hat{\mathbf{S}} \hat{\mathbf{x}}=\hat{\mathbf{g}}$. Two main versions were introduced in [10]: $(i)$ an exact reduction, in which $\hat{\mathbf{S}}$ is a block tridiagonal matrix, and (ii) an approximate reduction resulting in a block diagonal matrix $\hat{\mathbf{S}}$. These two approaches are briefly described next.

Basic SPIKE algorithm. The spikes $\mathbf{V}_{i}$ and $\mathbf{W}_{i}$, as well as the modified right-hand side and unknown vector partitions $\mathbf{g}_{i}$ and $\mathbf{x}_{i}$ can be further partitioned into their top and bottom $K$ rows and the middle $N_{i}-2 K$ rows, where $K$ is the half-bandwidth and $N_{i}$ is the size of partition $i$. For example, the three partitions of the vector $\mathbf{x}_{i}$ are $\mathbf{x}_{i}^{(t)}, \mathbf{x}_{i}^{\prime}$, and $\mathbf{x}_{i}^{(b)}$, respectively. With this, a block tridiagonal reduced system, $\hat{\mathbf{S}} \hat{\mathbf{x}}=\hat{\mathbf{g}}$ of dimension $2 K(P-1) \ll N$, is obtained by excluding the middle partitions of the spike matrices as:

$$
\left[\begin{array}{ccccc}
\mathbf{R}_{1} & \mathbf{M}_{1} & & & \\
& \ddots & & & \\
& \mathbf{N}_{i} & \mathbf{R}_{i} & \mathbf{M}_{i} & \\
& & & \ddots & \\
& & & \mathbf{N}_{P-1} & \mathbf{R}_{P-1}
\end{array}\right]\left[\begin{array}{c}
\hat{\mathbf{x}}_{1} \\
\vdots \\
\hat{\mathbf{x}}_{i} \\
\vdots \\
\hat{\mathbf{x}}_{P-1}
\end{array}\right]=\left[\begin{array}{c}
\hat{\mathbf{g}}_{1} \\
\vdots \\
\hat{\mathbf{g}}_{i} \\
\vdots \\
\hat{\mathbf{g}}_{P-1}
\end{array}\right]
$$


where

$$
\begin{aligned}
& \mathbf{N}_{i}=\left[\begin{array}{cc}
\mathbf{W}_{i}^{(b)} & \mathbf{0} \\
\mathbf{0} & \mathbf{0}
\end{array}\right], \quad i=2, \ldots, P-1 \\
& \mathbf{R}_{i}=\left[\begin{array}{cc}
I_{M} & \mathbf{V}_{i}^{(b)} \\
\mathbf{W}_{i+1}^{(t)} & I_{M}
\end{array}\right], \quad i=1, \ldots, P-1 \\
& \mathbf{M}_{i}=\left[\begin{array}{cc}
\mathbf{0} & \mathbf{0} \\
\mathbf{0} & \mathbf{V}_{k+1}^{(t)}
\end{array}\right], \quad i=1, \ldots, P-2
\end{aligned}
$$

and

$$
\hat{\mathbf{x}}_{i}=\left[\begin{array}{c}
\mathbf{x}_{i}^{(b)} \\
\mathbf{x}_{i+1}^{(t)}
\end{array}\right], \hat{\mathbf{g}}_{i}=\left[\begin{array}{c}
\mathbf{g}_{i}^{(b)} \\
\mathbf{g}_{i+1}^{(t)}
\end{array}\right], \quad i=1, \ldots, P-1
$$

The global solution $\mathbf{x}$ of the original system can then be reconstructed from $\hat{\mathbf{x}}$ using:

$$
\begin{aligned}
\mathbf{x}_{1}^{\prime} & =\mathbf{g}_{1}^{\prime}-\mathbf{V}_{1}^{\prime} \mathbf{x}_{2}^{(t)} \\
\mathbf{x}_{i}^{\prime} & =\mathbf{g}_{i}^{\prime}-\mathbf{V}_{i}^{\prime} \mathbf{x}_{i+1}^{(t)}-\mathbf{W}_{i}^{\prime} \mathbf{x}_{i-1}^{(b)}, \quad i=2, \ldots, P-1 \\
\mathbf{x}_{P}^{\prime} & =\mathbf{g}_{P}^{\prime}-\mathbf{W}_{P}^{\prime} \mathbf{x}_{P-1}^{(b)}
\end{aligned}
$$

Truncated SPIKE algorithm. When the banded matrix $\mathbf{A}$ is diagonally dominant, it can be shown that the elements of the left spikes $\mathbf{W}_{i}$ decay in magnitude from top to bottom, while those of the right spikes $\mathbf{V}_{i}$ decay from bottom to top [33]. This decay, which is more pronounced the larger the degree of diagonal dominance of $\mathbf{A}$, allows the approximation of the reduced matrix $\hat{\mathbf{S}}$ in Eq. (14) by its diagonal blocks only; i.e., setting the top and bottom parts of the right and left spikes, respectively, to zero. This results in the so-called truncated version of the SPIKE algorithm [10].

Each diagonal block $\mathbf{R}_{i} \hat{\mathbf{x}}_{i}=\hat{\mathbf{g}}_{i}$ of the resulting truncated reduced system can be solved independently using the following elimination steps ${ }^{\dagger}$ :

$$
\begin{gathered}
\text { Form } \overline{\mathbf{R}}_{i}=I_{M}-\mathbf{W}_{i+1}^{(t)} \mathbf{V}_{i}^{(b)} \\
\text { Solve } \overline{\mathbf{R}}_{i} \mathbf{x}_{i+1}^{(t)}=\mathbf{g}_{i+1}^{(t)}-\mathbf{W}_{i+1}^{(t)} \mathbf{g}_{i}^{(b)} \\
\text { Calculate } \mathbf{x}_{i}^{(b)}=\mathbf{g}_{i}^{(b)}-\mathbf{V}_{i}^{(b)} \mathbf{x}_{i+1}^{(t)}
\end{gathered}
$$

Since the spikes now contain parts that are never calculated explicitly, the global solution $\mathbf{x}$ cannot be retrieved any longer from the solution of the reduced system as in Eq. (17). Instead, the right-hand side $\mathbf{b}$ is first purified from the influences of the coupling blocks $\mathbf{B}_{i}$ and $\mathbf{C}_{i}$ :

$$
\begin{gathered}
\mathbf{A}_{1} \mathbf{x}_{1}+\left[\begin{array}{c}
\mathbf{0} \\
\mathbf{0} \\
\mathbf{B}_{1} \mathbf{x}_{2}^{(t)}
\end{array}\right]=\mathbf{b}_{1} \\
{\left[\begin{array}{c}
\mathbf{C}_{i} \mathbf{x}_{i-1}^{(b)} \\
\mathbf{0} \\
\mathbf{0}
\end{array}\right]+\mathbf{A}_{i} \mathbf{x}_{i}+\left[\begin{array}{c}
\mathbf{0} \\
\mathbf{0} \\
\mathbf{B}_{i} \mathbf{x}_{i+1}^{(t)}
\end{array}\right]=\mathbf{b}_{i}, \quad i=2, \ldots, P-1} \\
{\left[\begin{array}{c}
\mathbf{C}_{P} \mathbf{x}_{P-1}^{(b)} \\
\mathbf{0} \\
\mathbf{0}
\end{array}\right]+\mathbf{A}_{P} \mathbf{x}_{P}=\mathbf{b}_{P}}
\end{gathered}
$$

\footnotetext{
${ }^{\dagger}$ For simplicity, we keep the same notation for $\mathbf{x}_{i}^{(b)}$ and $\mathbf{x}_{i+1}^{(t)}$, even though they are now only approximations the solution of the tridiagonal reduced system of Eq. (14). 

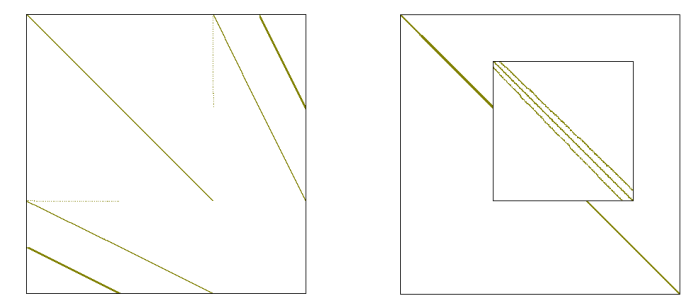

Figure 2. Sparsity pattern for the original (left) and reordered (right) matrix of dimension $N=88,950$. The sparsity pattern for a $2000 \times 2000$ diagonal block of the reordered matrix is displayed in the inset.

after which the global solution is obtained by solving the resulting block diagonal system using previously computed factorizations.

In addition to forming and factorizing a much smaller reduced matrix $\mathbf{R}$ than in the case of the basic SPIKE approach, additional efficiency gains can be obtained with the truncated algorithm by avoiding the calculation of the entire spike matrices. Indeed, using simultaneous LU and UL factorization of the diagonal blocks $\mathbf{A}_{i}$ the necessary $K \times K$ blocks $\mathbf{W}_{i}^{(t)}$ and $\mathbf{V}_{i}^{(b)}$ can be computed using truncated elimination sweeps of length $K$ only.

3.1.3. SPIKE as a preconditioner. As described above, if no drop-off is performed after reordering, the basic SPIKE algorithm represents a direct solution method for the original $\mathbf{A x}=\mathbf{b}$ system. Due to efficiency and memory limitations, this is not an attractive alternative for a parallel implementation, particularly on GPU cards which provide relatively small amounts of device memory. Alternatively, a solution that relies on the truncated SPIKE approach and possibly element drop-off requires an iterative solution refinement to account for these two approximations. This alternative turns SPIKE into a preconditioner. In this case, the diagonal dominance restriction can be relaxed and, using the reordering strategies described previously, a solver for general sparse linear systems can be constructed. Moreover, as described below and in section $\S 4.2$, additional approximations, both of the matrix and in the solution scheme, can be introduced to further improve efficiency.

Using the truncated SPIKE algorithm as a preconditioner for a Krylov-subspace iterative linear solver represents one of the options that the Spike: :GPU solver provides. However, Spike: : GPU implements additional strategies for further improvements in overall efficiency, in particular a second-stage reordering approach. Even though the combination MC64 RCM typically leads to significant bandwidth reduction, it is very common to obtain banded matrices with the band itself being very sparse. Notably, this is characteristic of the matrices encountered in multibody dynamics simulation. Consider for example the matrix shown on the left in Fig. 2, which has $N=88,950$ and on average only 5.78 non-zero elements per row. After reordering with no drop-off, the resulting banded matrix has a half-bandwidth $K=205$. However, as shown on the right in Fig. 2, the band itself is very sparse with a fill-in of only $0.7 \%$. Based on this observation, the default preconditioner in Spike: : GPU constructs a block banded matrix where each diagonal block (corresponding to one of the $P$ partitions of the banded matrix obtained after the first stage MC64 - RCM reordering) is allowed to have a different bandwidth. This is achieved using a second RCM pass carried out on each diagonal block separately and in parallel. Applying this strategy to the matrix in Fig. 2, using $P=16$ partitions, the equivalent half-bandwidth is reduced to $K=141$, while the fill-in of the band becomes $2.8 \%$.

It is important to note that, while the strategy of using a second stage reordering results in an overall efficiency improvement for all matrices we have tested, it cannot be used with the truncated SPIKE algorithm as described in the previous section. This is due to the fact that the RCM algorithm induces reorderings of the rows of the spike matrices $\mathbf{W}_{i}$ and $\mathbf{V}_{i}$ and therefore the assumptions justifying the truncated elimination sweeps are no longer met. As such, we revert to calculating the entire spike matrices using complete elimination sweeps from 
which we recover the necessary $K \times K$ blocks $\mathbf{W}_{i}^{(t)}$ and $\mathbf{V}_{i}^{(b)}$ after applying the appropriate inverse permutations. In this scenario, the computational bottleneck becomes the calculation of the full spike matrices which entails the solution of repeated multiple-RHS linear problems; this motivates some of the algorithmic decisions described in section $\S 4.2$.

3.1.4. The Spike::GPU library. Although this work discusses its use in conjunction with Chrono::Flex, Spike: :GPU is a stand-alone library that can be used for the solution of a given linear system or interfaced to other simulation packages.

Spike: :GPU is implemented as a templated C++ library using as template parameters matrix and vector types. For enhanced flexibility and portability, it makes use of the cusp [34] library for sparse linear algebra computations on GPUs and the thrust [35] library of parallel algorithms. Spike: :GPU provides a high-level interface which allows easily changing the floating point precision and sparse matrix format. In addition, the solver accepts a user-defined functor class to implement sparse matrix-vector product (SPMV) operations that defaults to an implementation based on the vectorized high performance cusp multiplication algorithm. Currently, Spike: : GPU provides access to some of the cusp Krylov-subspace iterative solvers (BiCGStab, GMRES, CG) and a custom implementation of BiCGStab $(\ell)$ [36]. For testing and comparison purposes, Spike: :GPU also provides an implementation of the MINRES [37] algorithm, although the underlying preconditioner is in general not appropriate for MINRES.

Some implementation details, peculiar to GPU computing, are provided in section $§ 4.2$. More details on the capabilities, structure, and implementation of Spike: : GPU can be found in [9]. The library is available open-source under a BSD license at [38].

\subsection{Using Spike::GPU in Chrono::Flex}

As long as no addition/deletion of bodies and bilateral constraints occur, the sparsity pattern of the Jacobian matrix $\mathbf{J}_{n+1}$ in Eq. (11) does not change during a simulation. Moreover, while its nonzero entries do change with the configuration of the system, their relative weights are mostly constant. Consequently, the reordering required by the SPIKE-based preconditioner can be performed only once, at the first simulation time step, and then reused whenever a preconditioner update is required. The preconditioner matrix is updated:

(a) upon starting a new simulation;

(b) when the average number of Krylov iterations per Newton iteration reaches a user-specified threshold;

(c) upon taking more than a user-specified integration steps since the last evaluation.

The above strategy attempts to balance the high cost of frequent Jacobian evaluations and factorizations with the slow convergence due to infrequent Jacobian updates. See section $\S 5.2$ for a study of various preconditioner update strategies.

The convergence, or stopping, test in the Newton iteration uses an absolute tolerance for the norm of the correction vector $\delta_{n+1}^{(k)}$ of Eq. (11). This tolerance is set, taking into account the Newmark update in Eq. (7a), to be $\epsilon_{N}=f \cdot \epsilon / h^{2}$, where $h$ is the integration step size, $\epsilon$ a user-specified precision level in positions, and $f$ a safety factor ${ }^{\ddagger}$.

Similarly, the errors in the iterative Krylov linear solver must also be controlled. In Spike: :GPU, the linear iteration error in the solution of $\mathbf{A x}=\mathbf{b}$ is controlled through the preconditioned residual vector, using a combination of relative and absolute tolerances. Specifically, we require that the preconditioned residual vector be less than $\epsilon_{K}^{r e l}\left\|\mathbf{r}_{0}\right\|+\epsilon_{K}^{a b s}$, where $\mathbf{r}_{0}=\mathbf{b}-\mathbf{A} \mathbf{x}_{0}$ is the initial residual; i.e., the residual corresponding to the initial guess $\mathbf{x}_{0}$. To ensure that the linear iteration errors do not interfere with the nonlinear error and integration accuracy, we set $\epsilon_{K}^{r e l}=\min \left\{0.01 \cdot \epsilon_{N}, 10^{-6}\right\}$ and $\epsilon_{K}^{a b s}=10^{-10}$.

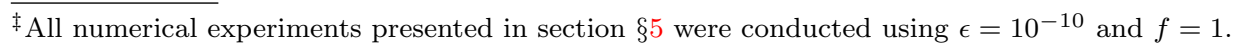


For additional efficiency gains, we take advantage of the flexibility provided by the templated implementation of Spike: :GPU and use a mixed-precision approach. All preconditionerrelated operations are performed in single precision floating-point arithmetic but the solution produced by the iterative linear solver is calculated in double precision.

\section{GPU PARALLEL IMPLEMENTATION}

GPU computing has been adopted in a wide spectrum of scientific and engineering data analysis, simulation, and visualization tasks. In fields as diverse as genomics, medical physics, astronomy, nuclear engineering, quantum chemistry, finance, oil and gas exploration, etc., GPU computing is attractive owing to its capacity to deliver speed-ups that in some cases can be as high as one order of magnitude compared to the execution on traditional multi-core CPUs. This performance boost is typically associated with computational tasks amenable to the single instruction multiple data (SIMD) processing paradigm. Modern GPUs have a deep memory hierarchy that at the low end displays bandwidths in excess of $300 \mathrm{~GB} / \mathrm{s}$ (global memory), while at the high-end, for shared memory and registers, displays low latencies and bandwidths in the 1-10 TB/s range. From a hardware architectural perspective, one of the salient features of GPUs is their ability to pack a very large number of rather unsophisticated scalar processors. These are organized in streaming multiprocessors (SMs), each GPU being the sum of several of these SMs that work independently by typically sharing less than 12 GB of main memory and $1 \mathrm{~GB}$ of L2 cache.

The presence of a deep memory hierarchy and numerous SMs turns GPU programming into a slightly more complicated task than CPU programming. In either case though, parallel computing brings forth unique aspects that control the efficiency of an implementation. An exhaustive discussion regarding the implementation of low level optimization techniques that concern the use of shared memory, global memory access patterns, minimization of data movement in memory and between the CPU and GPU falls outside the scope of this paper. Instead, we include high level software design decision that turned out to play a critical role in dictating the efficiency of Chrono: :Flex in general and Spike: :GPU in particular.

\subsection{Chrono::Flex implementation}

GPU computing excels at handling fine grain parallelism in conjunction with large problems that require minimal synchronization barriers. In the context of flexible multibody dynamics, this translates into using a larger set of generalized coordinates, as described in section $\S 2.1$, thus minimizing the coupling of ANCF nodal coordinates. With this, the assembly of the discretized EOM of Eq. 9 can be performed in parallel with no communication: GPU computing kernels for the calculation of internal forces are invoked with a number of threads equal to the number of ANCF elements in the system, while the calculation of the constraint equation residuals is performed in parallel over all constraint equations.

Matrix storage. The sparse matrices required to assemble the Jacobian of Eq. (11) are stored internally in Coordinate Format (COO). While a Compressed Sparse Row (CSR) format might be slightly more efficient for computing matrix-vector products, the $\mathrm{COO}$ format allows for a more efficient parallel assembly of the Jacobian matrix.

Sparse matrix-vector products. Except for the calculations performed in conjunction with the preconditioner, the main simulation cost in Chrono: : Flex is associated with the calculation of SPMV products in the iterative solver. For instance, BiCGStab(2) requires four such SPMV operations per iteration. We relied on a Spike: : GPU feature to provide a user-defined functor

class that implements a custom partitioned SPMV algorithm. Specifically, products of the form $\mathbf{w}=\mathbf{J} \mathbf{v}$ for a given vector $\mathbf{v}$ are calculated in two stages as

$$
\mathbf{w}=\left[\begin{array}{cc}
\mathbf{0} & \Phi_{\mathbf{q}}^{T} \\
\Phi_{\mathbf{q}} & \mathbf{0}
\end{array}\right] \mathbf{v}+\left[\begin{array}{cc}
\mathbf{M}+\mathbf{K}^{s} & \mathbf{0} \\
\mathbf{0} & \mathbf{0}
\end{array}\right] \mathbf{v}
$$


where we take advantage of the fact that for the formulation adopted in Chrono: : Flex the matrix in the first term on the right-hand side is constant throughout the simulation. The two terms are computed using the vectorized cusp multiplication algorithm.

\subsection{Spike::GPU implementation}

For more information on the various algorithmic details required for an efficient GPU implementation in Spike: :GPU we direct the reader to [9]. Here we only highlight some of the more relevant ones.

Number of partitions and partition size. Selection of the number of partitions $P$ must strike a balance between two conflicting requirements: workload of each block of GPU threads and accuracy of the truncated approximation. No attempt has been made to automate this selection and some experimentation is required. In the current implementation, for a given number of partitions $P$ and matrix dimension $N$, the partition sizes of the first $P_{r}$ partitions is $\lfloor N / P\rfloor+1$ while the remaining partitions are of size $\lfloor N / P\rfloor$. Note that $N=P\lfloor N / P\rfloor+P_{r}$.

Matrix storage. Spike: :GPU accepts any sparse matrix format supported by cusp. Internally, dense banded matrices are stored in arrays, logically structured as "tall-and-thin" matrices with $N$ rows and $2 K+1$ columns. This guarantees coalesced memory access in both the LU/UL factorization and the forward/backward elimination sweeps.

$L U / U L$ factorization methods. For problems where the half-bandwidth $K<64$, we adopt a so-called window sliding method using a single factorization kernel function with $\min \left(K^{2}, 512\right)$ parallel threads per block in order to reduce the overhead of GPU kernel launching, make better use of data cache, and eliminate the need for global thread synchronization. For $K>64$, we employ a block $L U$ factorization method, in which a fixed number of rows in the $\mathbf{U}_{i}$ matrices and columns in the $\mathbf{L}_{i}$ matrices are calculated simultaneously. This strategy offers a trade-off between parallelism and reducing the overhead of GPU kernel launching.

Diagonal boosting strategy. If the banded matrix obtained after reordering is diagonally dominant, the $\mathrm{LU}$ factorization of each diagonal block $\mathbf{A}_{i}$ can be done without pivoting. However, if the matrix is not diagonally dominant, one cannot guarantee that the diagonal blocks $\mathbf{A}_{i}$ are nonsingular. To ensure coalesced memory access, we continue to use LU factorizations of the diagonal blocks without pivoting but with diagonal boosting (perturbation). In this case, we obtain a factorization of a slightly perturbed diagonal block, $\mathbf{L}_{i} \mathbf{U}_{i}=\left(\mathbf{A}_{i}+\delta \mathbf{A}_{i}\right)$, where $\left\|\delta \mathbf{A}_{i}\right\|=\mathcal{O}(u\|\mathbf{A}\|)$ and $u$ is the unit roundoff [39].

Use of registers during elimination sweeps. As noted in section $§ 3.1 .3$, when using a second stage reordering strategy, the computational bottleneck in the preconditioner setup phase is in the solution of multiple-RHS linear systems needed for calculating the spike matrices. Considerable efficiency improvements can be obtained by partitioning the RHS vectors into small blocks so that all elements in the current block fit into high-bandwidth, low-latency registers.

Mixed-precision strategy. While in newer generation GPU accelerators, such as the NVIDIA Tesla K20 used for this work, the performance gap between single- and double-precision arithmetic has been significantly reduced [40], strategic use of precision in GPU calculations can have a substantial effect on overall performance. In our current implementation, we use a simple mixed-precision strategy by performing all SPIKE-related operations in single precision throughout the preconditioner stage and switching to double precision arithmetic in the outer Krylov solver calculations. Numerical experiments indicate that this strategy results in an average of $50 \%$ improvement in performance when compared with an approach where all calculations are performed in double precision. 


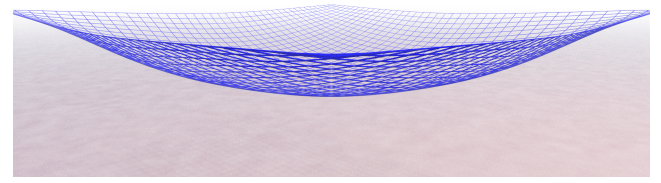

Figure 3. A net model composed of Chrono: : Flex beam elements.

\section{NUMERICAL RESULTS}

Using the Spike: :GPU solver in the context of Chrono::Flex results in an efficient Newton-Krylov solution of the underlying nonlinear problems with further benefits arising from the fact that the entire solution procedure is now completely implemented on the GPU.

We present the results of several different studies aimed first at gauging the effect of various algorithmic options and second at investigating the performance and scalability of the proposed approach. All these numerical experiments were conducted using a test problem consisting of a net composed of flexible beams as shown in Fig. 3. The model shown therein consists of 3,280 beam elements arranged in a $40 \times 40$ net, including 24,243 algebraic constraints, thus leading to a system of 63,603 nonlinear equations that must be solved at every time step. For the scaling analysis described in section 5.4 the size of the model was increased up to $80 \times 80$, corresponding to a problem in 252,003 unknowns.

Except where noted otherwise, all numerical experiments were carried out on an NVIDIA Tesla K20x GPU accelerator [40]. These cards feature a single GK110 Kepler GPU with 2688 CUDA cores and 6 GB GDDR5 memory.

\subsection{Comparison of Krylov solvers}

We begin with a discussion of the relative performance of various solvers on the $40 \times 40$ flexible net test problem (with a modulus of elasticity $E=2 \cdot 10^{7} \mathrm{~Pa}$ ). For the purposes of this study, we performed 5-second long simulations with a step size $h=10^{-3} \mathrm{~s}$ using the following solvers: unpreconditioned and preconditioned BiCGStab, unpreconditioned and preconditioned $\operatorname{BiCGStab}(2)$, and unpreconditioned MINRES.

The BiCGStab solver is included in this study to allow a comparison with the results presented in [5] which were obtained using an unpreconditioned BiCGStab solver. In many situations, in particular for the problems considered here, the linear system of Eq. (11) is a saddle point problem ${ }^{\S}$. We therefore include in this comparison the MINRES method [37] which was specifically designed to solve linear systems with symmetric, possibly indefinite matrices. In its standard form, MINRES can only be preconditioned with a positive definite preconditioner matrix. Unfortunately, for a saddle-point problem the resulting Spike: : GPU preconditioner is indefinite and thus not appropriate for preconditioning MINRES. While we plan on extending Spike: :GPU to support preconditioning of saddle point problems, here we only consider unpreconditioned MINRES.

Results are shown in Fig. 4. Taking the preconditioned BiCGStab(2) as the base case, the resulting speedups over unpreconditioned solvers, which held effectively constant across the entire simulation time range were as follows: 10.8 over BiCGStab - none, 8.3 over BiCGStab(2) - none, 3.6 over MINRES - none. We note that, although the results in Fig. 4 show the preconditioned BiCGStab as the most effective solver for this particular problem, we found

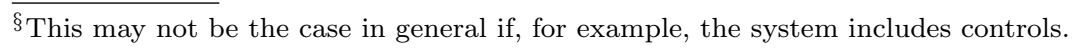




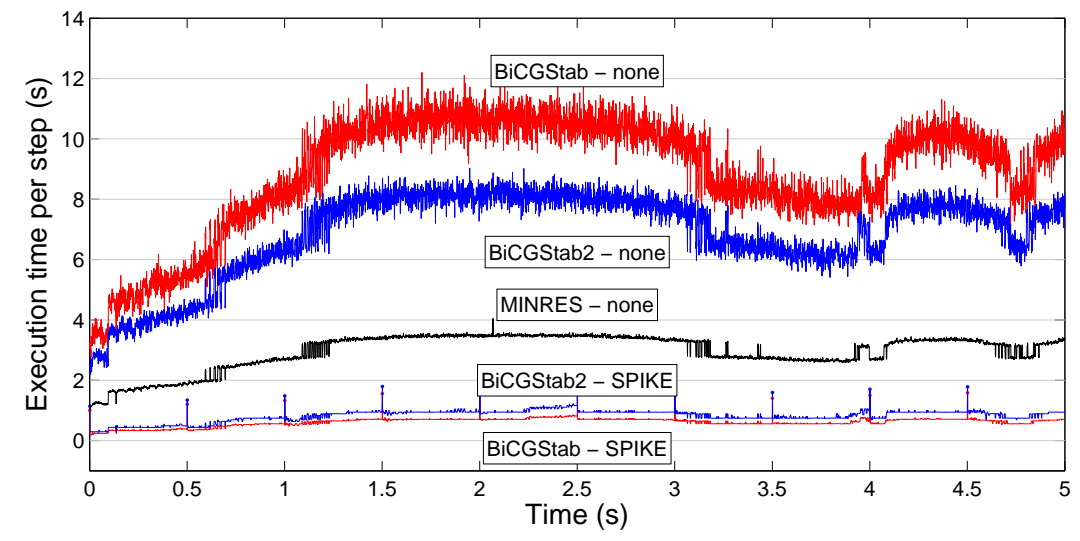

Figure 4. Timing comparison for various Krylov solvers, with or without preconditioning. The sharp increases in the curves for the two preconditioned solvers correspond to preconditioner updates which, for this study, were enforced every $0.5 \mathrm{~s}$. [ $40 \times 40$ net; $\left.E=2 \cdot 10^{7} \mathrm{~Pa} ; h=10^{-3} \mathrm{~s}\right]$
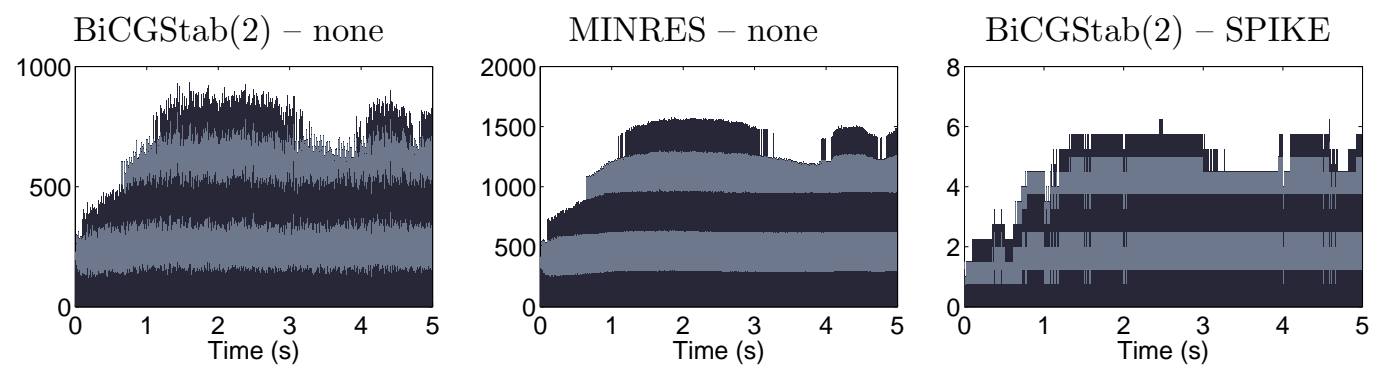

Figure 5. Number of Newton iterations and number of Krylov iterations per Newton iteration. Each colored band represents one Newton iteration, with the height of each band indicating the number of

Krylov linear iterations for that Newton iteration. [40 $\left.\times 40 \mathrm{net} ; E=2 \cdot 10^{7} \mathrm{~Pa} ; h=10^{-3} \mathrm{~s}\right]$

that it lacks overall robustness and often displays convergence stagnation. Therefore, in all subsequent experiments we restricted ourselves to using BiCGStab(2), both unpreconditioned and preconditioned, and (unpreconditioned) MINRES.

Figure 5 provides a breakdown of the number of Newton iterations required at each time step, as well as the number of Krylov iterations taken at each Newton iteration. As expected, at any given time step, all three solvers require the same number of Newton iterations, demonstrating that the tolerance used in the Krylov solver stopping criteria is appropriately set as not to interfere with convergence of the outer Newton loop. However, the number of Krylov iterations varies significantly for the three cases studied, with the preconditioned solver requiring only $1-2$ Krylov iterations for each linear system solution. Note also that, as the timing results in Fig. 4 show, the faster convergence rate of BiCGStab(2) does not offset the lower cost per iteration of MINRES (more on this in section $\S 5.4$ ).

As shown in Fig. (4), every once in a while, the amount of time required by the preconditioned solvers to find the solution increases. This occurs at the time steps at which a preconditioner update is enforced. While a preconditioner update reuses the reordering information cached at the start of the simulation (see section $§ 3.2$ ), it does require a factorization of the updated banded matrix. As discussed next, the factorization of the preconditioner matrix is about two to five times more costly than a typical preconditioner solve step. The infrequent factorizations in the proposed approach are a main reason for the efficiency of inexact Newton methods over strategies baed on direct linear solvers where the Jacobian $\mathbf{J}_{n+1}^{(k)}$ must be factored at each iteration and at each time step (classical Newton) or the Jacobian $\mathbf{J}_{n+1}^{(0)}$ must be factored once per time step (modified Newton). 
To gain a better understanding of the associated overhead, it is insightful to consider all the stages required to re-evaluate and factorize the Jacobian used as a preconditioner matrix. A re-evaluation of the $63,603 \times 63,603$ preconditioner matrix requires the computation of its 569,262 entries. Once these entries have been recomputed, the matrix is reordered. Producing the MC64 reordering takes $47 \mathrm{~ms}$ and the RCM reordering takes $149 \mathrm{~ms}$. Note that producing these two reorderings is carried out once, at the beginning of the simulation, using the Jacobian evaluated at the initial conditions, that is $\mathbf{J}_{1}^{(0)}$. The reordering information is passed to the GPU, in a process that takes less than $10 \mathrm{~ms}$, and stored in the GPU global memory. Any subsequent evaluation of the preconditioner matrix, which is carried out on the GPU, is going to rely on the reordering stored on the GPU and generated at the beginning of the simulation. The SPIKE strategy employed for this example called for the partitioning of the reordered banded matrix into $P=10$ sub-matrices (see section $\S 3.1 .2$ ). The amount of time required to produce the matrices $\mathbf{B}_{i}$ and $\mathbf{C}_{i}$ in Eq. (13) is $53 \mathrm{~ms}$. Carrying out the factorization of the 10 block matrices required $121 \mathrm{~ms}$. Computing the spikes $\mathbf{V}_{i}$ and $\mathbf{W}_{i}$ took $307 \mathrm{~ms}$, while the $\mathrm{LU}$ factorization of the reduced matrices $\mathbf{R}_{i}$ in Eq. (18) required $185 \mathrm{~ms}$. This brings the time required to compute a factorization of the preconditioner matrix to approximately $650 \mathrm{~ms}$. The cost of a single iteration of the preconditioned BiCGStab(2) algorithm is approximately $110 \mathrm{~ms}$. It is this last number that gives the amount of time required to find the solution of a linear system using the preconditioned Krylov solver.

\subsection{Comparison of preconditioner update strategies}

We investigate next the effect on overall performance of different preconditioner update strategies. As mentioned in section $\S 3.2$, the goal here is to strike a balance between the high relative cost of a preconditioner evaluation and the decreased convergence due to using out-of-date Jacobian information.

For this study, we use again the $40 \times 40$ flexible net, simulated over a 5 second interval using a step size $h=10^{-3} \mathrm{~s}$. Since the impact of an out-of-date preconditioner on the convergence rate of the BiCGStab(2) solver increases with problem stiffness, we use here a modulus of elasticity $E=2 \cdot 10^{8} \mathrm{~Pa}$. We note that the same trends, albeit less pronounced, are obtained a lower stiffnesses.

Figure 6 shows the execution time per time step for three different preconditioner update strategies:

1. No update, where the preconditioner was only evaluated at the initial time and kept fixed for the entire duration;

2. Uniform update, where a preconditioner update was enforced at uniformly-spaced time steps, in this case every 500 steps (or $0.5 \mathrm{~s}$ ).

3. Adaptive update, where a preconditioner update was triggered after any integration step that required more than 10 Krylov iterations, cumulative over all Newton iterations at that step.

Compared to the no update scenario, employing a uniform update strategy results in an overall speedup of 1.19, while adaptive update results in a speedup of 1.47 . We must note however that the selection of the various algorithmic parameters in the preconditioner update strategy is highly problem dependent and achieving optimal performance requires some experimentation.

\subsection{Effect of problem stiffness}

Results from a parametric study in which the modulus of elasticity was varied in the range $2 \cdot 10^{6} \mathrm{~Pa} \leq E \leq 2 \cdot 10^{11} \mathrm{~Pa}$ are summarized in Table I. The data represent execution times (in hours) for 1 second of simulation of the $40 \times 40$ flexible net, using a step size $h=10^{-4} \mathrm{~s}$. Results show that relative performance between the 3 solvers is maintained across all values of 


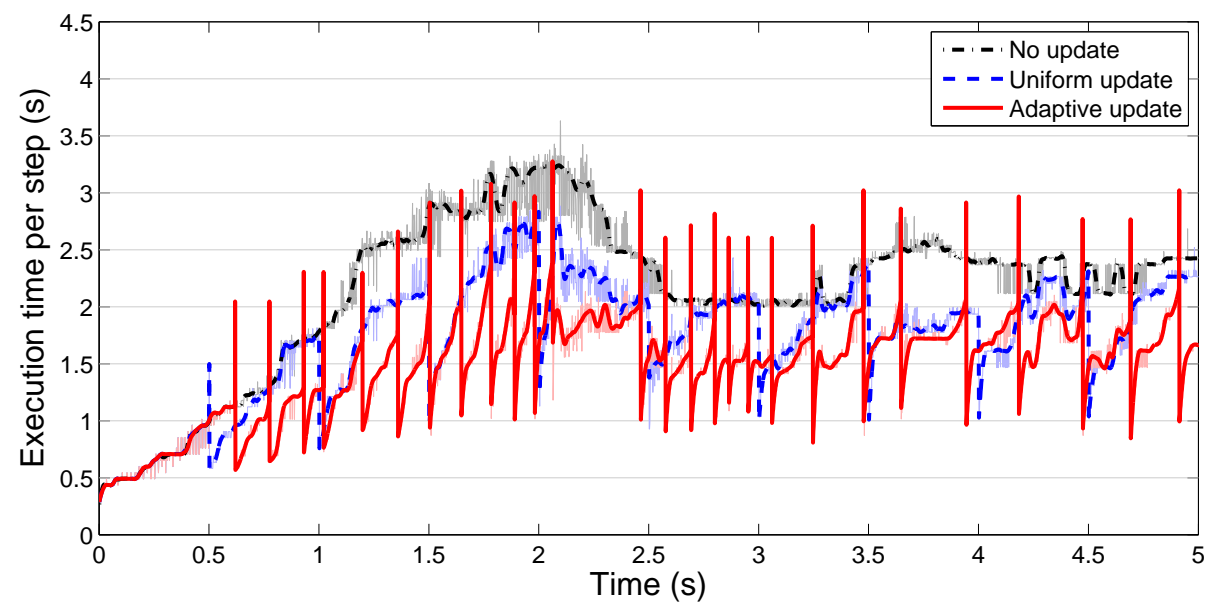

Figure 6. Comparison of different preconditioner update strategies for the preconditioned BiCGStab(2) solver. The solid lines in the foreground are filtered values of the raw measured timing data displayed in the background. [40 $\times 40$ net; $\left.E=2 \cdot 10^{8} \mathrm{~Pa} ; h=10^{-3} \mathrm{~s}\right]$

Table I. Execution time (in hours) for a 1-second simulation. A preconditioner update was enforced every $0.5 \mathrm{~s}$. $\left[40 \times 40\right.$ net; $\left.h=10^{-4} \mathrm{~s}\right]$

\begin{tabular}{l|rrrrrr}
\hline & \multicolumn{5}{|c}{ Elasticity $(\mathrm{Pa})$} \\
\multicolumn{1}{c|}{ Solver } & $2 \cdot 10^{6}$ & $2 \cdot 10^{7}$ & $2 \cdot 10^{8}$ & $2 \cdot 10^{9}$ & $2 \cdot 10^{10}$ & $2 \cdot 10^{11}$ \\
\hline BiCGStab(2) - none & 8.29 & 8.18 & 7.80 & 7.53 & 8.51 & 13.74 \\
MINRES - none & 3.62 & 3.58 & 3.51 & 3.41 & 3.65 & 6.55 \\
BiCGStab(2) - SPIKE & 0.91 & 0.94 & 0.97 & 0.84 & 1.09 & 1.48 \\
\hline
\end{tabular}

the modulus of elasticity, with preconditioned BiCGStab(2) being about 8 times faster than unpreconditioned BiCGStab(2) and about 4 times faster than unpreconditioned MINRES.

As seen from the data in Table I, there is little variation in execution times across almost the entire modulus of elasticity range. This can be easily explained with the plots in Fig. 7 which illustrate the dependency of the number of Newton and Krylov iterations on the integration step size and problem stiffness. While we only provide these plots for the preconditioned BiCGStab(2) solver, similar trends were observed for the other two solvers considered herein. These results simply show that the integration step size used to generate the data in Table I was too conservative; as a consequence, a single Krylov iteration per Newton iteration is required for convergence for most values of $E$ considered. However, in the absence of an adaptive step size algorithm (one of the planned future extensions of Chrono: : Flex) and given that the focus of the present paper is on studying the effect of preconditioning, rather than experimenting with different integration step sizes at different levels of stiffness, we decided to use a common value of $h$, small enough to ensure stable integration of all problems in the range of interest.

\subsection{Effect of problem size}

As a final numerical experiment, we performed a series of simulations of net models of increasing size, to estimate how the solver performance scales with problem dimension. Unlike the numerical experiments described so far, for this study we used an NVIDIA Tesla K40c GPU accelerator which offers 12 GB of memory thus allowing simulations of net sizes of up to $80 \times 80$ (i.e., a problem dimension of 252,003 ).

Table II lists relevant size information for the models considered in this experiment, including the number of equations $N$, the number of non-zero elements $n n z$ in the sparse Jacobian, and information on the distribution of non-zero elements on each row of the Jacobian. The last two columns provide preconditioner-specific information, namely the half-bandwidth $K$ obtained 

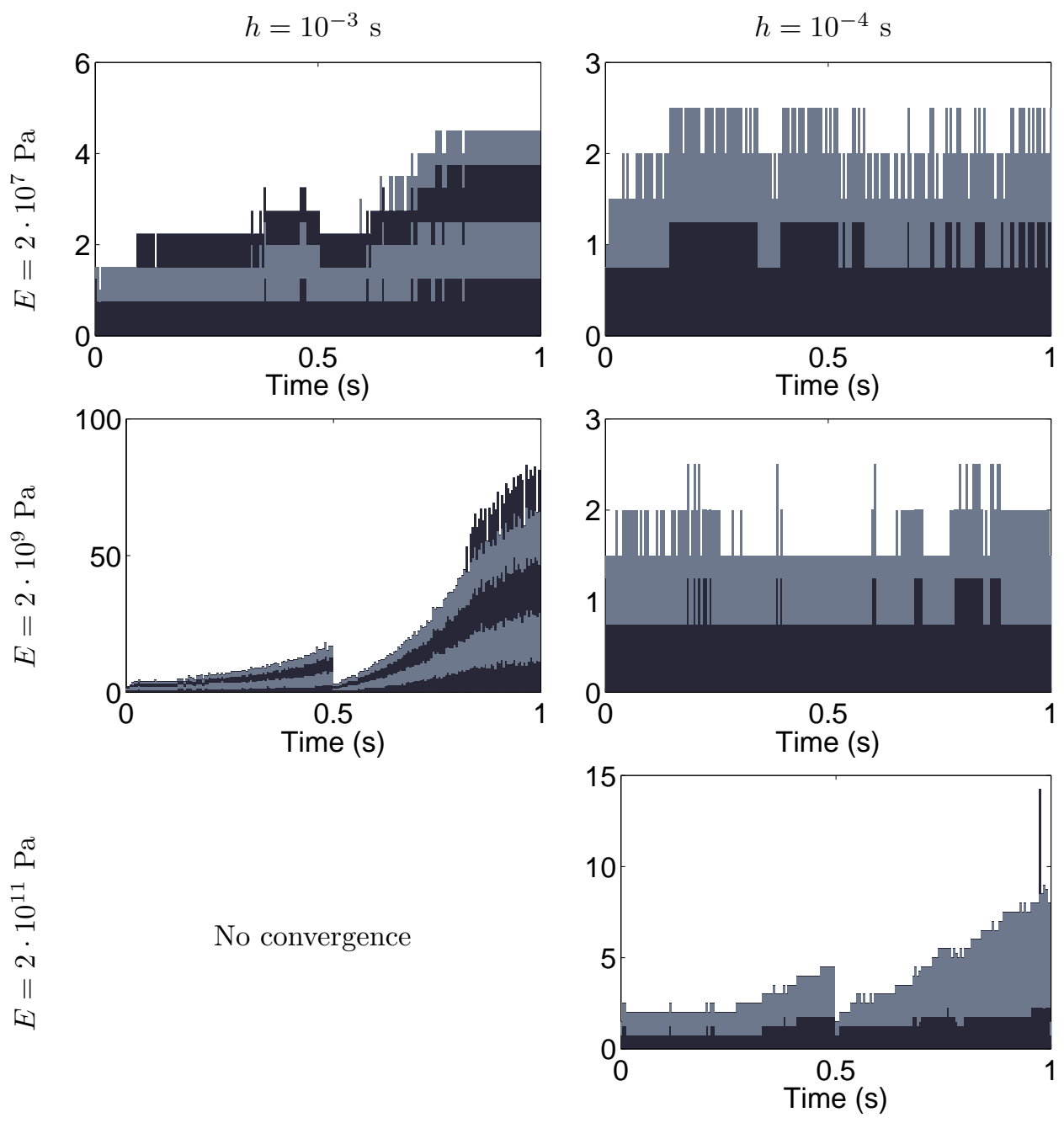

Figure 7. Number of Krylov iterations per Newton iteration for the preconditioned BiCGStab(2) solver, as function of modulus of elasticity $E$ and integration step size $h$. [40 $\times 40$ net]

Table II. Problem dimensions for net models of different sizes

\begin{tabular}{r|rr|rrrr|rr}
\hline \multirow{2}{*}{ Net size } & & & \multicolumn{7}{|c}{ nnz per row } & & \\
\hline $40 \times 40$ & 63,603 & 569,262 & 8.950 & 1 & 14 & 5.467 & 852 & 10 \\
$50 \times 50$ & 99,003 & 885,582 & 8.945 & 1 & 14 & 5.470 & 1062 & 11 \\
$60 \times 60$ & 142,203 & $1,271,502$ & 8.942 & 1 & 14 & 5.472 & 1272 & 12 \\
$70 \times 70$ & 193,203 & $1,727,022$ & 8.939 & 1 & 14 & 5.474 & 1482 & 17 \\
$80 \times 80$ & 252,003 & $2,252,142$ & 8.937 & 1 & 14 & 5.475 & 1694 & 13 \\
\hline
\end{tabular}

after the first-stage reordering (see section §3.1.1) and the optimal number of partitions $P$, the latter obtained through experimentation.

Timing results for 1-second long simulations of flexible nets with $E=2 \cdot 10^{7} \mathrm{~Pa}$ and increasing dimensions are provided in Fig. 8. In all cases, the integration step size was $h=10^{-3} \mathrm{~s}$. The fact that all three solvers exhibit linear time complexity may be surprising at first glance and deserves a more detailed explanation.

Consider first the two unpreconditioned Krylov solvers, labeled BiCGStab(2) - none and MINRES - none in Fig. 8. Excluding the SPMV operations, all operations performed during one iteration of either Krylov method (dot products, axpy, vector copies, etc.) have $\mathcal{O}(N)$ 


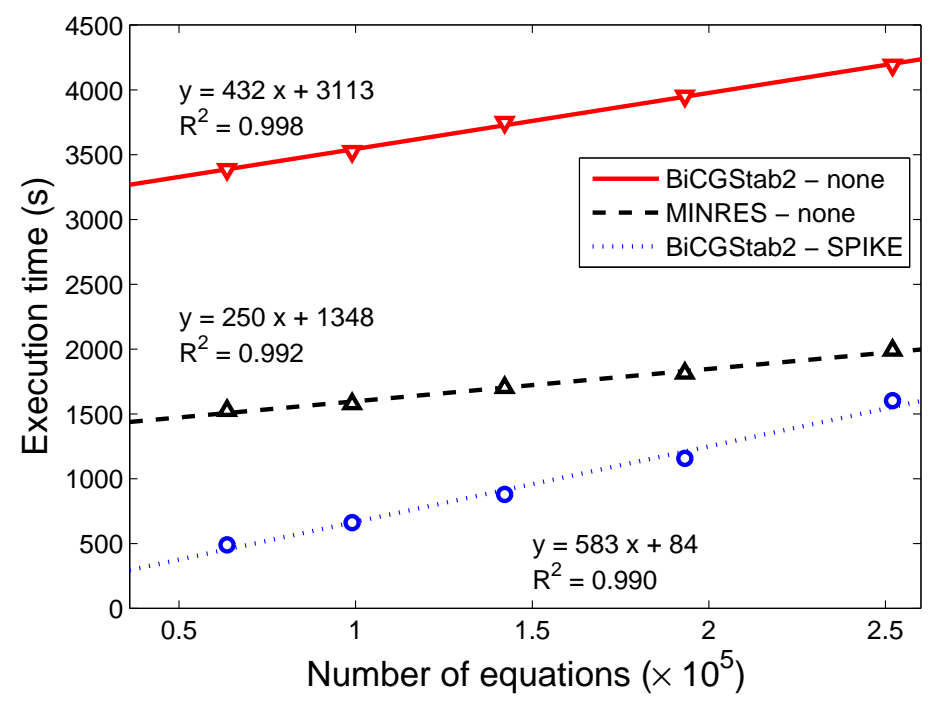

Figure 8. Execution time for 1-second simulations as function of problem size and solver type. $\left[E=2 \cdot 10^{7} \mathrm{~Pa} ; h=10^{-3} \mathrm{~s}\right]$

complexity. The special structure of the Jacobian matrices arising in the problems considered here is such that the number of non-zero elements per row is independent of the problem size, as shown in Table II. This results in linear complexity of the SPMV operations and therefore a cost of an unpreconditioned Krylov iteration that scales linearly with problem size. Although not shown here, the trends shown in Fig. 5 for the $40 \times 40$ net hold unchanged over the entire range of problem dimensions considered. In particular, MINRES requires approximatively 1.83 times more Krylov iterations than BiCGStab(2). However, each iteration of the unpreconditioned BiCGStab(2) algorithm is approximately 4 times as expensive as a MINRES iteration (counting for example the number of SPMV operations). These observations are in perfect agreement with the observed overall relative speedup of about 2.2 of MINRES over unpreconditioned BiCGStab(2) (see Fig. 8).

When using a preconditioned Krylov method, each iteration incurs additional costs related to the one or more preconditioner solve operations (typically equal in number with the number of SPMV operations) $)^{\pi}$. For the Spike: :GPU preconditioner, the complexity of a preconditioner solve operation is dictated by the cost of the required forward and backward elimination sweeps for a given partitioned LU factorization. In general, for a banded matrix of size $N$ and bandwidth $K$ the elimination sweeps are $\mathcal{O}(K N)$ and the data presented in Table II, which indicates that $K \propto \sqrt{N}$, therefore suggests an overall complexity of the preconditioned BiCGStab(2) solver of $\mathcal{O}(N \sqrt{N})$. However, using the second-stage reordering strategy described in section $\S 3.1 .3$ coupled with experimentally-determined optimal number of partitions, leads to block banded partitions with half-bandwidths $K_{i}$ that are practically independent of the problem size. This results in a preconditioned BiCGStab(2) linear solver that has linear time complexity for the problems arising in implicit integration of flexible multibody dynamics.

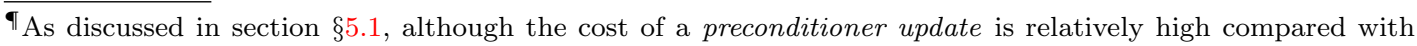
that of a preconditioner solve operation, these updates are very infrequent and their cost quickly amortized over the length of a simulation.
} 


\section{CONCLUSIONS AND FUTURE WORK}

This contribution describes a flexible multibody dynamics solution methodology that is entirely mapped onto the GPU for parallel simulation of large compliant systems. Although illustrated here in the context of the ANCF method, we expect the findings reported herein to be independent of the specific formalism adopted for the formulation of the EOMs. Assuming that an implicit integration formula is used to discretize the resulting index 3 DAEs of multibody dynamics, the solution methodology proposed relies on a preconditioned NewtonKrylov technique to solve the discretized EOM. The main contribution of this paper is the implementation and use of a preconditioned parallel sparse solver for the solution of the linear systems encountered in the inner Newton-Krylov loop. The preconditioning is shown to be responsible for an eight-fold reduction in run times when simulating large problems with thousands of flexible beam elements.

We would like to note that, for the problems considered here, MINRES is a very attractive alternative, especially as the problem size increases. Unfortunately, the Spike: :GPU preconditioner cannot be used with MINRES when solving saddle-point problems, since standard MINRES requires a positive definite preconditioner. However, recent results [41] indicate that a projected MINRES method is well defined when applied to saddle-point problems with an (indefinite) constraint preconditioner. We plan on investigating how the Spike: : GPU methodology can be extended to support such preconditioners.

A future investigation avenue will focus on assessing the effectiveness of spectral reordering methods which could be very attractive given that the sparsity pattern of $\mathbf{J}_{n+1}^{(k)}$ remains fixed during the entire simulation. It is generally accepted that a spectral method [42], based on the Fiedler vector" [43], outperforms the RCM algorithm in reducing the profile of large sparse matrices.

On the Chrono::Flex front, we intend to implement variable step strategies, like the one described in [21], that adapt the integration step size to reflect the transients of the underlying dynamics. Additionally, for greater flexibility and modeling power, we plan on extending Chrono: :Flex to support additional ANCF elements, besides gradient deficient beam elements.

Finally, it remains to understand and gauge the software implementation effort and simulation efficiency trade-offs related to moving from GPU to parallel multi-core CPU computing on architectures such as the 12-core (24 virtual) Intel CPUs or the 60-core Intel Xeon Phi accelerator.

\section{ACKNOWLEDGEMENTS}

Financial support has been provided in part by a US Army TARDEC ARO grant under Contract No. W911NF-11-D-0001-0107, National Science Foundation grant SI2-SSE-1147337, and the Army Research Office instrumentation grant W911NF-11-1-0327.

\section{REFERENCES}

1. Hairer E, Wanner G. Solving Ordinary Differential Equations II: Stiff and Differential-Algebraic Problems. Springer, 1996.

2. Atkinson KE. An Introduction to Numerical Analysis. Second edn., John Wiley \& Sons Inc.: New York, 1989.

3. Saad Y. Iterative methods for sparse linear systems. Society for Industrial and Applied Mathematics, 2003.

4. Brown PN, Hindmarsh AC, Petzold LR. Using Krylov methods in the solution of large-scale differentialalgebraic systems. SIAM J. Sci. Comput. 1994; 15:1467-1488.

\footnotetext{
"The Fiedler vector is the eigenvector corresponding to the smallest non-trivial eigenvalue of the Laplacian matrix associated with the connectivity graph of $\mathbf{J}_{n+1}^{(k)}$
} 
5. Melanz D, Khude N, Jayakumar P, Negrut D. A matrix-free Newton-Krylov parallel implicit implementation of the Absolute Nodal Coordinate Formulation. ASME Journal of Computational and Nonlinear Dynamics 2013; 9(1):011 006, doi:10.1115/1.4025281.

6. Khude N, Stanciulescu I, Melanz D, Negrut D. Efficient parallel simulation of large flexible body systems with multiple contacts. ASME Journal of Computational and Nonlinear Dynamics 2013; 8(4):041 003041003.

7. Mazhar H, Heyn T, Pazouki A, Melanz D, Seidl A, Bartholomew A, Tasora A, Negrut D. Chrono: a parallel multi-physics library for rigid-body, flexible-body, and fluid dynamics. Mechanical Sciences 2013; 4(1):49-64.

8. Project Chrono. Chrono: An Open Source Framework for the Physics-Based Simulation of Dynamic Systems 2014. URL http://www.projectchrono.org/.

9. Li A, Serban R, Negrut D. A SPIKE-based approach for the parallel solution of sparse linear systems on GPU cards. Technical Report TR-2013-05, SBEL, University of Wisconsin - Madison 2013.

10. Polizzi E, Sameh A. A parallel hybrid banded system solver: the SPIKE algorithm. Parallel Computing 2006; 32(2):177-194.

11. Bauchau O. Flexible Multibody Dynamics. Solid mechanics and its applications, Springer, 2010. URL http://books.google.com/books?id=Pn94Czlg7VUC.

12. Berzeri M, Campanelli M, Shabana AA. Definition of the elastic forces in the finite-element absolute nodal coordinate formulation and the floating frame of reference formulation. Multibody System Dynamics 2001; 5:21-54.

13. von Dombrowski S. Analysis of large flexible body deformation in multibody systems using absolute coordinates. Multibody System Dynamics 2002; 8:409-432. URL http://dx.doi.org/10.1023/A: $1021158911536,10.1023 / \mathrm{A}: 1021158911536$.

14. Gerstmayr J, Shabana A. Analysis of thin beams and cables using the absolute nodal co-ordinate formulation. Nonlinear Dynamics 2006; 45(1):109-130.

15. Shabana AA. Computational Continuum Mechanics. First edn., Cambridge University Press, 2008.

16. Shabana AA. Dynamics of Multibody Systems. Third edn., Cambridge University Press, 2005.

17. Johnson KL. Contact mechanics. Cambridge University Press, 1987.

18. Haug EJ. Computer-Aided Kinematics and Dynamics of Mechanical Systems Volume-I. Prentice-Hall: Englewood Cliffs, New Jersey, 1989.

19. Negrut D, Jay L, Khude N. A discussion of low-order numerical integration formulas for rigid and flexible multibody dynamics. Journal of Computational and Nonlinear Dynamics 2009; 4:021 008-1.

20. Newmark NM. A method of computation for structural dynamics. Journal of the Engineering Mechanics Division, ASCE 1959; :67-94.

21. Negrut D, Rampalli R, Ottarsson G, Sajdak A. On an implementation of the Hilber-Hughes-Taylor method in the context of index 3 differential-algebraic equations of multibody dynamics (detc2005-85096). J. Comput. Nonlinear Dyn 2007; 2(1):73-85.

22. Hussein B, Negrut D, Shabana A. Implicit and explicit integration in the solution of the absolute nodal coordinate differential/algebraic equations. Nonlinear Dynamics 2008; 54(4):283-296.

23. Arnold M, Bruls O. Convergence of the generalized- $\alpha$ scheme for constrained mechanical systems. Technical Report 9-2007, Martin Luther University, Fachbereich Mathematik und Informatik, Halle-Wittenberg 2007.

24. Bottasso CL, Bauchau OA, Cardona A. Time-step-size-independent conditioning and sensitivity to perturbations in the numerical solution of index three differential algebraic equations. SIAM Journal on Scientific Computing 2007; 3:395-420.

25. Saad Y. Iterative methods for sparse linear systems. Society for Industrial and Applied Mathematics, 2003.

26. Sameh A, Kuck D. On stable parallel linear system solvers. JACM 1978; 25(1):81-91.

27. Polizzi E, Sameh A. SPIKE: A parallel environment for solving banded linear systems. Computers $E_{3}$ Fluids 2007; 36(1):113 - 120 .

28. Duff I. Algorithm 575: Permutations for a zero-free diagonal [F1]. ACM Transactions on Mathematical Software (TOMS) 1981; $7(3): 387-390$.

29. Duff I, Koster J. The design and use of algorithms for permuting large entries to the diagonal of sparse matrices. SIAM J. Matrix Analysis and Applications 1999; 20(4):889-901.

30. Duff I, Koster J. On algorithms for permuting large entries to the diagonal of a sparse matrix. SIAM J. Matrix Analysis and Applications 2001; 22(4):973.

31. HSL: A collection of Fortran codes for large-scale scientific computation. http://www.cse.clrc.ac.uk/nag/hsl 2011.

32. Cuthill E, McKee J. Reducing the bandwidth of sparse symmetric matrices. Proceedings of the 24th ACM Conference, New York, 1969; 157-172.

33. Mikkelsen C, Manguoglu M. Analysis of the truncated SPIKE algorithm. SIAM J. Matrix Analysis Applications 2008; 30(4):1500-1519.

34. Bell N, Garland M. Cusp: Generic parallel algorithms for sparse matrix and graph computations 2012. URL http://cusplibrary.github.io/, version 0.3.0.

35. Hoberock J, Bell N. Thrust: A parallel template library 2010. URL http://thrust.github.io/, version 1.7.0.

36. Sleijpen G, Fokkema D. BiCGStab(l) for linear equations involving unsymmetric matrices with complex spectrum. Electornic Transactions on Numerical Analysis 1993; 1:11-32.

37. Paige C, Saunders M. Solution of sparse indefinite systems of linear equations. SIAM J. on Numerical Analysis 1975; 12(4):617-629.

38. Spike::GPU 2013. URL https://github.com/spikegpu/SpikeLibrary.

39. Manguoglu M, Sameh A, Schenk O. PSPIKE: A parallel hybrid sparse linear system solver. Proceedings of the 15th International Euro-Par Conference on Parallel Processing, Springer-Verlag: Delft, The 
Netherlands, 2009; 797-808.

40. NVIDIA TESLA KEPLER GPU accelerators 2012. URL http://www.nvidia.com/content/ tesla/pdf/Tesla-KSeries-Overview-LR.pdf.

41. Gould N, Orban D, Rees T. Projected Krylov Methods for Saddle-Point Systems. Technical Report G2013-23, Cahier du GERAD, Ecole Polytechnique, Montreal, Canada 2013.

42. Barnard S, Pothen A, Simon H. A spectral algorithm for envelope reduction of sparse matrices. Numerical Linear Algebra with Applications Jul-Aug 1995; 2(4):317-334, doi:\{10.1002/nla.1680020402\}.

43. Fiedler M. Algebraic connectivity of graphs. Czechoslovak Mathematical Journal 1973; 23:298-305. 